La publicité qui reste: usages et réactualisations des artefacts publicitaires du passé

\title{
La bande-annonce : un artefact publicitaire réactualisé par les internautes. Le cas de Star Wars sur Youtube
}

Movie Trailers: When Internauts Reuse an Advertising Artifact. The Case of Star

Wars on Youtube

Stéphanie Marty

\section{CpenEdition}

Journals

Édition électronique

URL : http://journals.openedition.org/edc/7990

DOI : $10.4000 /$ edc.7990

ISSN : 2101-0366

Éditeur

Université de Lille

Édition imprimée

Date de publication : 1 décembre 2018

Pagination : 53-78

ISBN : 978-2-917562-20-8

ISSN : $1270-684$

Référence électronique

Stéphanie Marty, «La bande-annonce : un artefact publicitaire réactualisé par les internautes. Le cas de Star Wars sur Youtube », Études de communication [En ligne], 51 | 2018, mis en ligne le 01 janvier 2021, consulté le 11 février 2021. URL : http://journals.openedition.org/edc/7990 ; DOI : https:// doi.org/10.4000/edc.7990 


\section{La bande-annonce :}

un artefact publicitaire réactualisé par les internautes.

Le cas de Star Wars sur Youtube

Movie Trailers: When Internauts Reuse an Advertising Artifact. The Case of Star Wars on Youtube 
Cet article met au jour différentes formes de réactualisations d'anciennes bandes-annonces de la saga Star Wars, impulsées par les Youtubeurs. Ces réactualisations révèlent un nouveau rapport (facilité, décomplexé, transgressif) des publics contemporains aux artefacts publicitaires anciens. Elles révèlent également combien le numérique transforme les artefacts publicitaires, leur nature (poreuse, jouable) et leur carrière (étendue et prolongée par les internautes). En outre, ces réactualisations de bandes-annonces du passé rappellent le poids des objets du passé dans nos sociétés contemporaines, et le caractère stratégique de la nostalgie, qui semble constituer, aujourd'hui, une opportune ressource marketing. Enfin, ces réactualisations de bandes-annonces soulignent le potentiel des communautés en ligne, aujourd'hui capables de bricoler des artefacts du passé et d'étendre, ainsi, leur existence, jusque dans le présent de nos sociétés.

Mots-clés : bande-annonce, numérique, Youtubeurs, réactualisations.
This article explores the contemporary reuse and recycling of Star Wars movie trailers as practiced by todays' Youtubers. The study of these recontextualized trailers reveals new relationships between contemporary publics and advertising artifacts. Recontextualized and reworked movie trailers illustrate how digital technology transforms advertising artifacts, their nature (porous, pliable), and their lifespan (extended by web surfers). Reused and retooled trailers remind us as well of the weight of objects from the past in modern societies, and of the strategic nature of nostalgia, a fertile marketing resource. Finally, these reworked movie trailers highlight the potential of online communities, now able to "poach" advertising artifacts and to extend their existence into present-day societies.

Keywords: movie trailer, digital technology, Youtubers, recontextualization, reuse. 
Rétro, vintage, old school... Les réactualisations d'objets du passé semblent s'immiscer chaque jour davantage dans la société contemporaine (Reynolds, 2012). Dans cet article, nous proposons d'interroger les réactualisations d'artefacts publicitaires d'autrefois et, plus précisément, les réactualisations de bandes-annonces anciennes. Dans un premier temps, nous évoquons les travaux académiques qui traitent des réactualisations contemporaines de produits et d'artefacts publicitaires anciens, sans pourtant questionner les réactualisations contemporaines d'artefacts publicitaires cinématographiques anciens tels que les affiches ou les bandes-annonces. Dans un deuxième temps, nous présentons la méthodologie que nous avons mise en œuvre dans notre recherche portant sur un corpus de vidéos qui réactualisent des bandes-annonces de la saga Star Wars et qui sont diffusées par les internautes sur Youtube. Enfin, la dernière partie de cet article fait état des résultats qui émergent de notre analyse. Elle présente une typologie des réactualisations des bandes-annonces de Star Wars disponibles sur Youtube et examine les enjeux de ces réactualisations.

\section{1. \\ Artefacts publicitaires du passé et réactualisations contemporaines}

Afin d'initier notre propos, nous nous penchons sur les travaux académiques abordant les réactualisations contemporaines de produits et d'artefacts publicitaires anciens. Cette démarche nous conduit à constater que peu de recherches se concentrent sur les réactualisations contemporaines d'artefacts publicitaires cinématographiques anciens.

\subsection{Artefacts publicitaires et réactualisations}

La société aime fouiller dans les coffres du passé et remettre l'ancien au goût du jour (Courbières, 2016). On retrouve notamment cette dynamique dans le secteur publicitaire (Berthelot-Guiet, 2013). En effet, de nombreux remaniements de produits anciens voient le jour aujourd'hui : en témoignent notamment la réapparition de pots de Danette vintage, de la marinière Petit Bâteau, de la basket Stan Smith d'Adidas ou encore de la Mini Austin. Mais cet engouement pour le revival ne concerne pas exclusivement les emballages et les produits : il touche également les artefacts publicitaires. En effet, une multitude d'affiches (Chevry-Pébayle et De Iulio, 2015), de spots audiovisuels, d'objets publicitaires anciens ont traversé les époques et ont été remis en circulation par des amateurs, des collectionneurs, des professionnels, entreprenant des projets de collection, des opérations de restauration, de sauvegarde. 
1.2. Cinéma, artefacts publicitaires passés, numérique : quelles réactualisations ?

Dans le secteur cinématographique, de nombreux artefacts publicitaires sont conçus pour promouvoir les films (Croquet, 1998 ; Laurichesse, 2009 ; Chirouze, 2007 ; Duchet, 2009) : affiches, teasers, bandes-annonces. Au fil du temps, le numérique a bousculé la façon dont ces artefacts publicitaires sont réalisés et diffusés. En effet, à l'ère du numérique, ces différents artefacts sont désormais réalisés via des techniques numériques élaborées et déployés au moyen de campagnes transmedia (Bourdaa, 2013), invitant les internautes à intervenir et à participer (Charest et al., 2013). Ainsi, le numérique ne transforme pas uniquement la façon dont ces artefacts publicitaires sont réalisés et déployés. Il transforme également la façon dont ils sont reçus par les publics. En effet, le numérique donne aujourd'hui aux publics l'opportunité de s'emparer de ces artefacts publicitaires et des internautes profitent de cette opportunité pour réinventer et réactualiser les artefacts publicitaires cinématographiques. Ces pratiques font l'objet de recherches (Williams, 2010 ; Hartwig, 2012 ; Deaville, 2016) qui restent pourtant minoritaires. En effet, dans la littérature scientifique, les travaux sont majoritairement consacrés à la morphologie des pratiques promotionnelles déployées autour des films et aux pratiques cinématographiques liées au choix de film. Peu d'auteurs questionnent la façon dont les publics réactualisent les artefacts publicitaires cinématographiques. De même, peu d'enquêtes interrogent les formes hétérogènes que peuvent prendre ces réactualisations et les enjeux qu'elles peuvent introduire dans les pratiques de publics et dans les stratégies de la promotion cinématographique. Cet article se propose de fournir des éléments de réponse à ces questions et d'étudier les réactualisations d'artefacts publicitaires cinématographiques anciens initiées par les internautes : les formes que peuvent prendre ces réactualisations et les enjeux qu'elles peuvent porter.

\section{2. \\ Focus sur des artefacts publicitaires passés et réactualisés : les bandes-annonces de la saga Star Wars}

Afin d'étudier les pratiques de réactualisation des artefacts publicitaires cinématographiques, nous avons tout d'abord choisi de focaliser notre attention sur les réactualisations de bandes-annonces de la saga Star Wars diffusées sur la plateforme Youtube. Pour ce faire, nous avons d'abord saisi, sur Youtube, un certain nombre de mots clés : "bande-annonce Star Wars " et «trailer Star Wars ". Puis, à partir des vidéos obtenues en résultat, nous avons relevé, par des captures d'écran, les vidéos publiées par des internautes et contribuant à réactualiser des bandes-annonces de Star Wars. Nous avons ainsi rassemblé 107 captures d'écran, constituant un corpus nous permettant d'explorer notre 
objet de recherche. Nous avons analysé ce corpus à partir d'une analyse de contenu, veillant à identifier des formes communes, à dégager progressivement différentes formes de réactualisations de bandes-annonces initiées par les internautes sur Youtube et à construire une typologie. Afin d'interroger les enjeux portés par ces réactualisations, nous avons confronté à plusieurs reprises les émergences de cette analyse à des études et des questionnements plus vastes sur le rapport des sociétés contemporaines avec le passé.

\subsection{Les bandes-annonces en tant qu'artefacts publicitaires}

Le choix de nous pencher tout particulièrement sur les réactualisations contemporaines de ces artefacts publicitaires bien particuliers que sont les bandes-annonces est lié à plusieurs raisons.

D'une part, nous considérons que les bandes-annonces constituent de véritables artefacts publicitaires (Lozano Delmar, 2012). En effet, elles font partie des dispositifs promotionnels utilisés dans les campagnes publicitaires (Ferrandi, Kruger, 2009) orchestrées pour inciter les publics à aller voir un film (Euzeby, Martinez, 2004). Leur diffusion est d'ailleurs précédée de projections-tests, visant à étudier la façon dont les publics les reçoivent et les accueillent. Afin de séduire les publics, les bandes-annonces suivent à la fois les principes du marketing expérientiel (Marion, 2003), car elles donnent une sensation de l'expérience d'un produit, en l'occurrence, ici, d'un film, et les principes du teasing (Cochoy, 2011), car elles visent à attiser la curiosité des publics sans dévoiler complétement le contenu du film. Diverses études rappellent toutefois que les bandes-annonces ne maîtrisent qu'en partie les principes de ces deux stratégies marketing. En effet, certaines ${ }^{1}$ indiquent que les bandes-annonces n'offrent pas toujours un " échantillon expérientiel » du film qu'elles promeuvent, mais tendent vers une uniformisation croissante, marquée par la mise en avant des meilleurs moments du film (gags, actions), l'utilisation d'une voix off et de phrases d'accroches (épithètes élogieux sur le film) et la présentation systématique des stars et des logos de producteurs. D'autres études soulignent que les bandes-annonces ne maîtrisent pas toujours l'art du teasing et dévoilent parfois trop d'éléments ${ }^{2}$ au point que $36 \%$ des Français préfèrent ne pas regarder les bandes-annonces afin de découvrir le film en salles ${ }^{3}$ et que certaines instances comme la Motion Pictures Association of America instaurent progressivement des règles pour

1 Daniel Bô, Structure et rhétorique des bandes-annonces de films. Disponible sur : http://testconso.typepad.com/semiologie/2008/10/structure-et-rh.html (page consultée le $1^{\text {er }}$ octobre 2018).

2 Cf. l'étude réalisée par le cabinet Quali-Quanti, CNC Les affiches et les bandes annonces des films, décembre 2000. Disponible sur : http://www.qualiquanti. com/wp-content/uploads/2014/05/affetbafilmsword.pdf (page consultée le $1^{\text {er }}$ octobre 2018).

3 Étude réalisée par GroupM France, sur un échantillon représentatif de 699 répondants, âgés de 16 à 65 ans, entre le 31 octobre et le 9 novembre 2017, via un questionnaire en ligne. 
limiter leur durée. Au-delà de ces remarques, les bandes-annonces restent des artefacts publicitaires qui font partie à plein titre des outils du marketing de la culture et de la promotion des œuvres culturelles (Bourgeon-Renault, 2009 ; Beuscart, Mellet, 2012).

D'autre part, nous avons choisi de nous concentrer sur les bandes-annonces, car elles constituent un type d'artefacts publicitaires qui a traversé les époques. En effet, celles-ci existent depuis le début des années 1910 et ont connu un certain nombre de transformations. D'abord diffusées en fin de films pour annoncer le prochain épisode - d'où, en anglais, le terme trailer, signifiant littéralement caravane - elles sont aujourd'hui déployées en amont de tous les films et annoncent leur sortie dans les salles de cinéma, à la télévision, à la radio (en version audio), sur le web. Autrefois prises en charge aux EtatsUnis par la National Screen Service, société créée dans les années 1920 pour produire et distribuer les bandes-annonces, elles sont aujourd'hui conçues et réalisées selon les stratégies cross-médias des studios, qui contribuent à en faire un genre en soi.

Enfin, nous avons choisi d'étudier les bandes-annonces car celles-ci, qu'elles soient contemporaines ou anciennes, sont des artefacts audiovisuels particulièrement manipulés, aujourd'hui, par les internautes. En effet, en ligne, les internautes commentent, partagent, font circuler, publient de nouvelles versions de bandes-annonces (récentes ou anciennes). Ainsi, les bandes-annonces constituent un point d'entrée privilégié pour étudier les réactualisations d'artefacts publicitaires du passé.

\subsection{Les bandes-annonces de la saga Star Wars}

Nous avons choisi de nous concentrer sur les réactualisations des bandes-annonces d'une saga cinématographique en particulier : Star Wars. En effet, par plusieurs aspects, cette saga constitue, à notre sens, un matériau particulièrement fertile pour notre étude.

L'une des raisons qui nous a conduit à nous pencher sur la saga Star Wars est sa longévité. En effet, Star Wars est une saga de huit opus, sortis entre 1977 et 2017 : Star Wars IV Un nouvel espoir (Lucas, 1977) ; Star Wars V L'empire contre-attaque (Kershner, 1980) ; Star Wars VI Le retour du Jedi (Marquant, 1983) ; Star Wars I La menace fantôme (Lucas, 1999); Star Wars II L'attaque des clônes (Lucas, 2002) ; Star Wars III la revanche des Sith (Lucas, 2005) ; Stars Wars VII Le réveil de la force (Abrams, 2015) ; Star Wars VIII Les derniers Jedi (Johnson, 2017). La longévité de cette saga, étendue sur quarante années, offre donc l'opportunité d'étudier ce que deviennent les bandes-annonces anciennes (et plus largement les artefacts publicitaires anciens), notamment depuis l'arrivée du numérique.

Outre ce phénomène de longévité, nous avons choisi de nous concentrer sur les bandes-annonces de Star Wars car il s'agit d'une saga états-unienne 
mais produite et distribuée à l'échelle mondiale et devenue au fil du temps une référence culturelle internationale. En effet, Star Wars est un "film franchise ", une série qui a mobilisé, depuis ses débuts et jusqu'à aujourd’hui, un grand nombre d'individus et a donné lieu à la naissance d'une grande communauté internationale de fans, en s'appuyant progressivement sur un modèle transmédia, dans lequel les passionnés contribuent via des récits, des productions, des créations, à l'extension de l'univers de la saga (Jenkins, 2002). Ainsi, Star Wars fait aujourd'hui l'objet de la créativité d'un grand nombre d'individus (Jenkins, 2002) qui publient sur la toile un tissu foisonnant de productions en lien avec la saga ou avec les dispositifs qui la promeuvent, les bandes-annonces notamment. Tout à la fois ancrée dans le passé (en vertu de sa longévité) et dans le présent (en vertu des productions contemporaines que lui dédient les internautes), la saga Star Wars conjugue ancienneté et contemporanéité et offre une opportunité de saisir des pratiques contemporaines centrées sur la réactualisation d'artefacts publicitaires anciens. Pour toutes ces raisons, Star Wars nous semble donc constituer un point d'entrée pertinent pour étudier comment les internautes réactualisent aujourd'hui les bandes-annonces.

\subsection{La plateforme Youtube comme terrain de recherche}

Afin d'étudier les réactualisations des bandes-annonces de la saga Star Wars impulsées aujourd'hui par les internautes, nous avons décidé de nous focaliser sur la plateforme d’hébergement de vidéos créée en 2005, Youtube.

Première raison : notre étude cherche à saisir la façon dont les internautes réactualisent les bandes-annonces. Dans la mesure où les bandes-annonces sont des artefacts publicitaires vidéo, la plateforme Youtube nous semble être un terrain fertile, car elle constitue précisément un point de rencontre entre les internautes et les contenus vidéo. En effet, en 2014, elle est connue par $93 \%$ des Français ${ }^{4}$. En 2016, un Français sur deux regarde des vidéos sur Youtube tous les jours 5 . En 2017, Youtube compte 21127000 vidéonautes uniques et 1283676000 vidéos vues par mois ${ }^{6}$. Ainsi, la plateforme Youtube constitue un point d'intersection entre de nombreux internautes et de nombreuses vidéos, et notamment des bandes-annonces.

Deuxième raison : nous avons choisi d'étudier la façon dont certaines bandes-annonces anciennes (en l'occurrence, celles de la saga Star Wars) sont réactualisées par les internautes. Sur cet aspect aussi, Youtube nous semble constituer un terrain fécond. En effet, cette plateforme donne aux internautes

4 Enquête Ipsos-Steria, réalisée par internet du 14 au 19 mai 2014 auprès d'un échantillon de 1005 personnes (échantillon national représentatif de la population française âgée de 15 ans et plus).

5 Enquête Connected Consumer Study 2016 - TNS Infratest - Base : français âgés de 16 à 44 ans.

6 Enquête Mediametrie/NetRatings - Audience Vidéo - Tous lieux de connexion - France - octobre 2017 - Base : 2 ans et plus. 
l'opportunité de visionner, mais également de publier et de partager des vidéos. Aussi, sur Youtube, les internautes peuvent se procurer des vidéos (et notamment des bandes-annonces) contemporaines ou anciennes et les re-publier dans une nouvelle version. Ainsi, la plateforme Youtube constitue un point de rencontre entre les bandes-annonces et les publics et offre aux publics une multitude d'opportunités pour le visionnage, le téléchargement et la remise en circulation de bandes-annonces. Elle s'avère en ce sens particulièrement pertinente pour notre étude.

\subsection{La constitution d'un corpus de bandes-annonces réactualisées}

Afin de saisir les réactualisations des bandes-annonces de Star Wars émergeant sur Youtube, nous avons constitué un corpus. Pour cela, nous avons défini un certain nombre de mots clés que nous avons saisis sur Youtube dans le champ réservé aux requêtes. La première requête que nous avons choisi de saisir est " bande-annonce Star Wars ", car nous souhaitions appréhender les formes de réactualisations qui se développent précisément autour de cet artefact publicitaire (la bande-annonce) et autour de cette saga cinématographique (Star Wars). Nous avons choisi de ne pas traduire le nom de la saga en français (en saisissant la requête "bande-annonce Guerre des étoiles ") car Star Wars est à la fois le nom original et officiel de la saga et de la licence, mais également le nom repris massivement par des publics de tout pays. Si nous n'avons pas choisi de traduire le terme Star Wars en français, nous avons en revanche choisi de traduire le terme bande-annonce en anglais. En effet, la deuxième requête que nous avons choisi de saisir est "trailer Star Wars ", le terme trailer (bande-annonce en anglais) nous permettant de répondre à notre objectif (embrasser la diversité, l'hétérogénéité et l'épaisseur des pratiques de réactualisations de bandes-annonces de Star Wars) en intégrant une dimension internationale et sans se limiter aux réactualisations initiées par les Youtubeurs français. Par ailleurs, nous avons choisi de ne pas ajouter, dans nos requêtes, des chiffres romains ou arabes (« bande annonce Star Wars 1 » ou « trailer Star Wars /I "), afin de ne pas nous limiter aux réactualisations de certains opus de la saga, mais d'appréhender au contraire, dans leur diversité, les réactualisations des différents trailers de Star Wars (et notamment les réactualisations des trailers anciens).

Progressivement, les requêtes " bande-annonce Star Wars " et " trailer Star Wars " nous ont permis d'accéder à un volume considérable de contenus publiés, par les internautes, sur Youtube, autour des bandes-annonces de Star Wars. Face à ce volume de contenus, nous avons entrepris un travail d'exploration et de sélection : nous avons exploré et prélevé, au moyen de captures d'écran, les contenus qui concouraient à réactualiser les différentes bandes-annonces de Star Wars. Nous avons archivé ces captures d'écran sur des diapositives, dans un fichier Powerpoint. Au total, nous avons rassemblé 107 captures d'écran (provenant de 107 vidéos différentes) introduisant, selon nous, des formes de réactualisations des bandes-annonces de Star Wars. Nous 
avons entrepris un travail d'analyse de ces 107 captures d'écran, au moyen d'une analyse de contenu thématique.

\subsection{Analyse de contenu : les enjeux des portés par les réactualisations}

Afin d'analyser le corpus, nous avons procédé à une analyse passant par deux démarches complémentaires.

Première démarche : nous avons soumis le corpus à une analyse de contenu thématique (Bardin, 2013). Nous avons notamment cherché à identifier, parmi les captures d'écran, des similarités et des différences, à extraire des catégories, voire des formes communes. Cette démarche nous a permis de dégager progressivement différentes formes de réactualisations des bandes-annonces de Star Wars initiées par les internautes sur Youtube.

Deuxième démarche : nous avons confronté les éléments émergeant de l'analyse de contenu thématique à certaines tendances mises en évidence par les études sur le rapport des sociétés contemporaines avec le passé. Nous avons ainsi tenté de montrer dans quelle mesure les pratiques des internautes autour des bandes-annonces cinématographiques anciennes se relient à de nouvelles façons de se rapporter aux objets d'un temps désormais éloigné. Cette démarche nous a permis d'interroger les enjeux portés par les réactualisations de bandes-annonces initiées par les internautes sur Youtube et de rapprocher donc notre travail empirique à des questionnements plus vastes.

Ces deux démarches nous ont permis, d'une part, d'entrevoir la façon dont les artefacts publicitaires (en l'occurrence les bandes-annonces de Star Wars) sont aujourd'hui réactualisés par les internautes. Elles nous ont conduit en outre à nous questionner sur les enjeux et les implications de ces réactualisations.

\section{3. \\ Formes et enjeux des réactualisations de bandes-annonces relevées sur Youtube}

Sur Youtube, les internautes exploitent aujourd'hui les possibilités offertes par le numérique pour donner vie à différentes formes de réactualisations des bandes-annonces de la saga Star Wars. Dans cette section, nous pointerons différentes formes de réactualisations et nous réfléchirons aux enjeux portés par ces revitalisations.

3.1. $(\mathrm{Re})$ mise en circulation de bandes-annonces par les internautes : un rapport facilité (et facilitateur) à des artefacts publicitaires poreux?

Certains internautes choisissent de réactualiser d'anciennes bandes-annonces de Star Wars en assurant leur circulation sans les transformer. Certains se les procurent et les mettent en ligne sur Youtube : leur pratique repose 
alors sur une (re)mise en circulation de l'artefact publicitaire dans sa version originale. Dans la figure $n^{\circ} 1$, la bande-annonce de Star Wars // est publiée sans y apporter aucune modification.

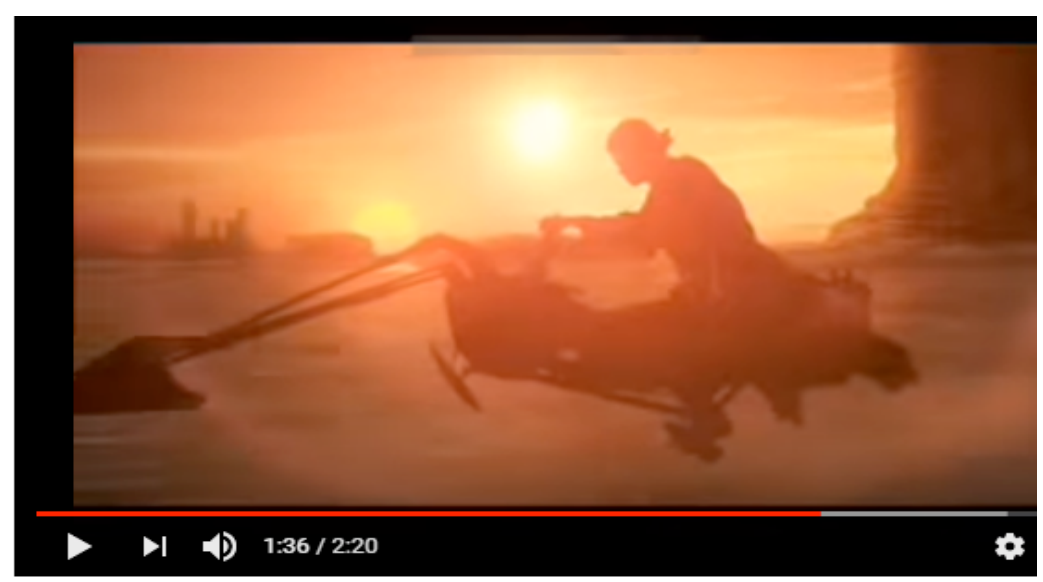

Star Wars, Episode 2: Attack of the Clones Trailer

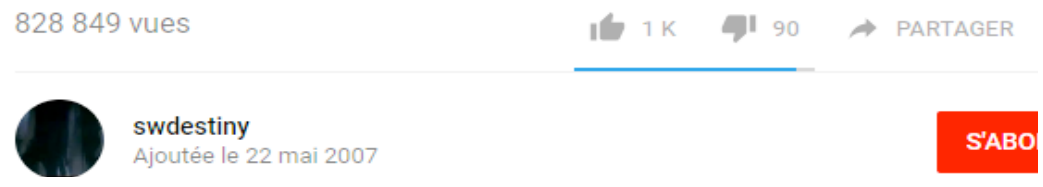

Figure 1 : Réactualisation de bandes-annonces de Star Wars par des remises en circulation ${ }^{7}$

D'autres internautes utilisent d'anciennes bandes-annonces de Star Wars dans leur version originale, mais les juxtaposent à d'autres contenus et notamment à d'autres bandes-annonces de la saga. Ainsi, des compilations, des classements (Top 10, Top 5) des bandes-annonces de Star Wars fleurissent sur Youtube. Dans la capture d'écran ci-dessous, plusieurs bandes-annonces de Star Wars sont ainsi réactualisées simultanément, à travers une compilation. 


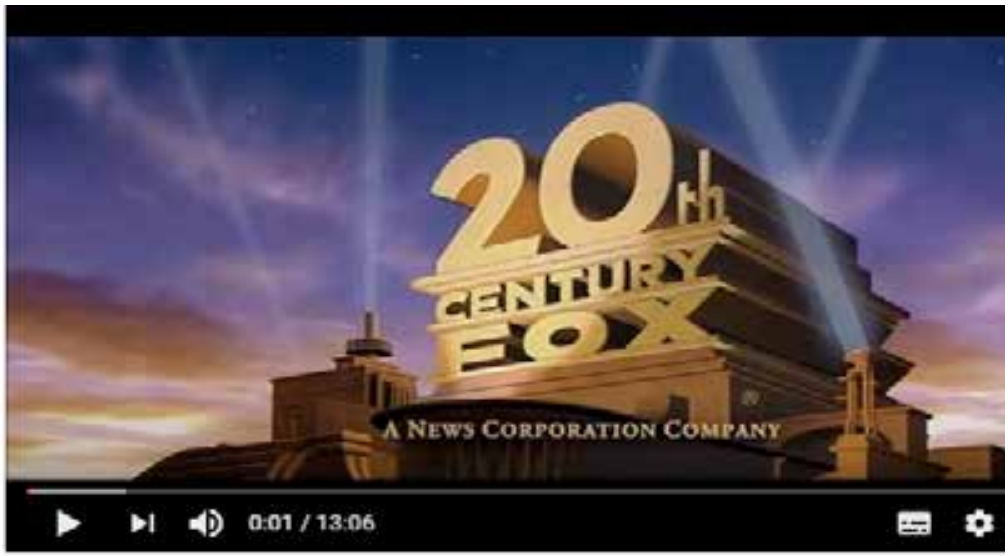

Star Wars Episode 1, 2, 3, 4, 5, 6 \& 7 Trailers HD (1977 - 2015).

Figure 2 : Réactualisation de bandes-annonces de Star Wars par des compilations ${ }^{8}$

Si certains réactualisent des bandes-annonces de Star Wars en créant des compilations, d'autres les réactualisent en les confrontant à des bandes-annonces d'autres sagas ou d'autres films (dans la dynamique des battles). Par exemple, dans la capture d'écran $n^{\circ} 3$, un internaute réactualise la bande-annonce de Star Wars en la confrontant à l'une des bandes-annonces de la saga Avengers. 


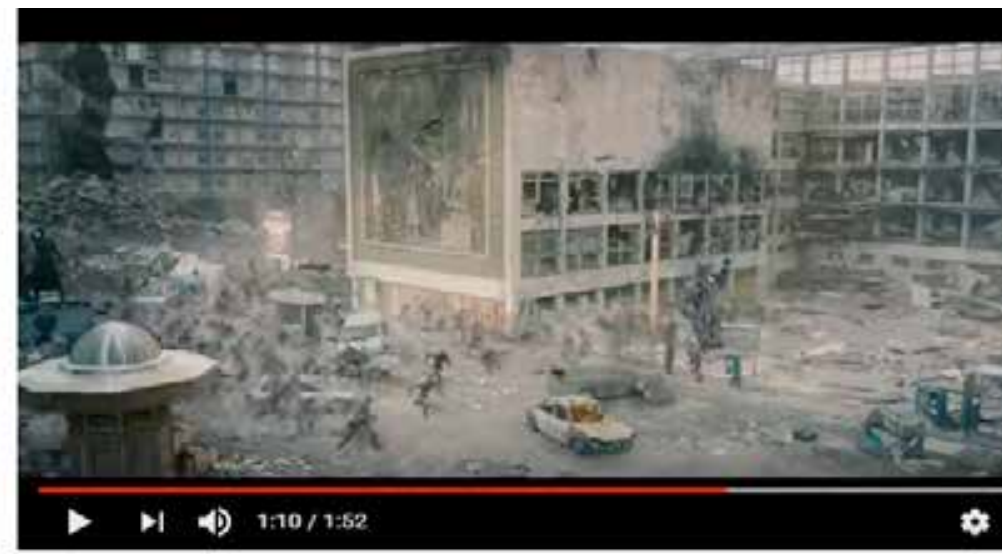

Star Wars VS Avengers Trailer

22144 vues

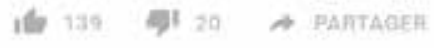

Zanyviper

A fouteve le 16 avr, 2015

Figure 3 : Réactualisation de bandes-annonces de Star Wars par des battles ${ }^{9}$

D'autres remettent en circulation des bandes-annonces de Star Wars en les juxtaposant à des vidéos de face cam reactions (c'est-à-dire des vidéos de réaction face à une caméra, en l'occurrence face à une webcam), dans lesquelles ils livrent des réactions personnelles. La capture d'écran présente dans la figure $n^{\circ} 4$ illustre ce type de juxtaposition. 


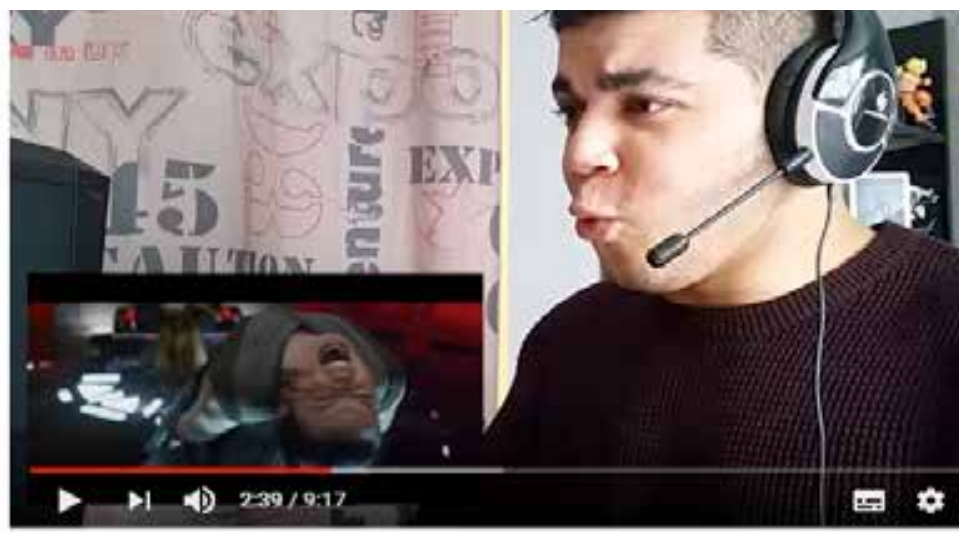

Star Wars The Last Jedi Trailer | REACTION FR !!!!!!

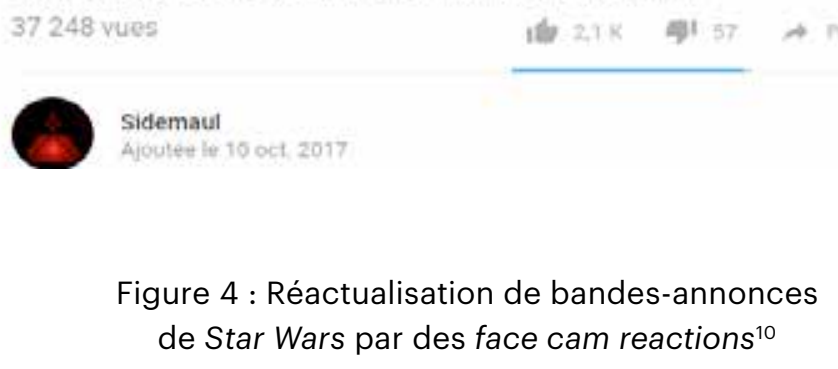

Ces différentes pratiques révèlent combien les internautes jouissent, à l'ère du numérique, d'une possibilité d'accès à des contenus sans précédent (Gayraud, Heuguet, 2015) et d'un rapport à la fois facilité et facilitateur à certains artefacts publicitaires anciens. En effet, avant le numérique, il était particulièrement difficile de repérer des bandes-annonces déployées quarante ans plus tôt. A l'ère du numérique, les bandes-annonces s'avèrent bien plus accessibles et bien plus poreuses. En effet, les internautes se procurent d'anciennes bandes-annonces (rapport facilité) et assurent leur circulation (Tabary-Bolka, 2009), garantissant simultanément leur accessibilité à d'autres internautes (rapport facilitateur). Ces différentes formes de réactualisations de bandes-annonces du passé révèlent combien les pratiques relevées sur Youtube sont proches de ce que Jeanneret (2014) appelle la « trivialité ». En effet, à travers leurs réactualisations de bandes-annonces, les internautes se montrent capables de donner lieu sur Youtube à une circulation créative d'objets culturels. 
3.2. Transformation de bandes-annonces par les internautes : un rapport transgressif aux artefacts publicitaires?

Certains internautes ne se contentent pas de réactualiser les bandes-annonces de Star Wars en les (re)mettant en circulation. Ils les réactualisent en les transformant, sur le plan audio ou visuel. Sur le plan audio, ils changent notamment la bande-son, la musique ou le doublage dans la dynamique de doublage par les fans (fandubbing). La figure $n^{\circ} 5$ en est un exemple.

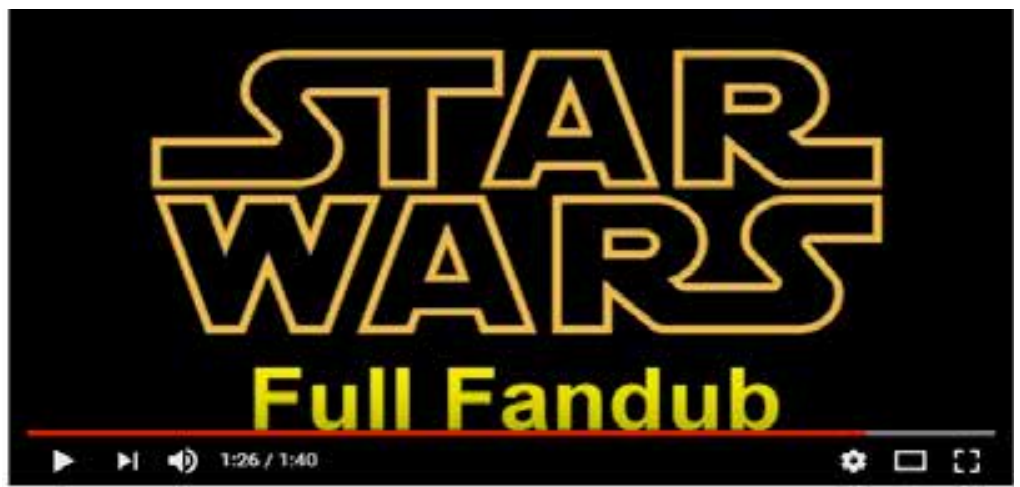

Star Wars: A New Hope Full Fandub Trailer! 307 vues

ShadowGlambert

Figure 5 : Réactualisation de bandes-annonces de la saga Star Wars par des fandubs ${ }^{11}$

Les internautes réactualisent parfois les bandes-annonces de la saga Star Wars en les transformant sur le plan visuel. La figure $n^{\circ} 6$ illustre la façon dont les internautes réactualisent la bande-annonce de Star Wars VII en prenant en charge le sous-titrage, dans la dynamique du fansubbing (sous-titrage par les fans). 


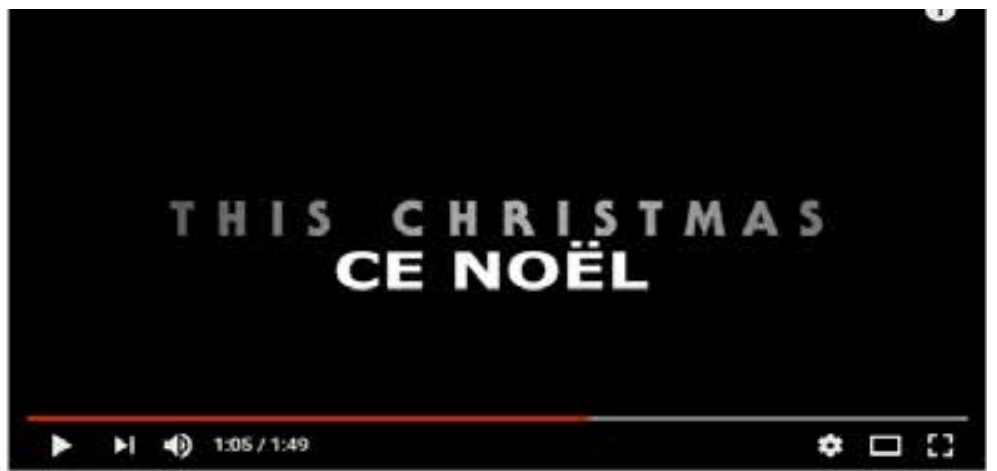

Star Wars VII Bande-annonce Trailer $\$ 2$ VOSTFR Sous-titré français

6178 vees

G GeekLandMaster French

Figure 6 : Réactualisation de bandes-annonces de la saga Star Wars par des fansubs ${ }^{12}$

Toujours sur le plan visuel, certains internautes intègrent - dans des bandes-annonces de Star Wars - des extraits d'autres bandes-annonces : dans la dynamique du mash up, ils créent des contenus composites, en combinant des matériaux audiovisuels différents. La figure $n^{\circ} 7$ montre un mash up créé par un internaute, combinant des images de Star Wars /l et des images de la bande-annonce du film Garden State (Braff, 2004). 


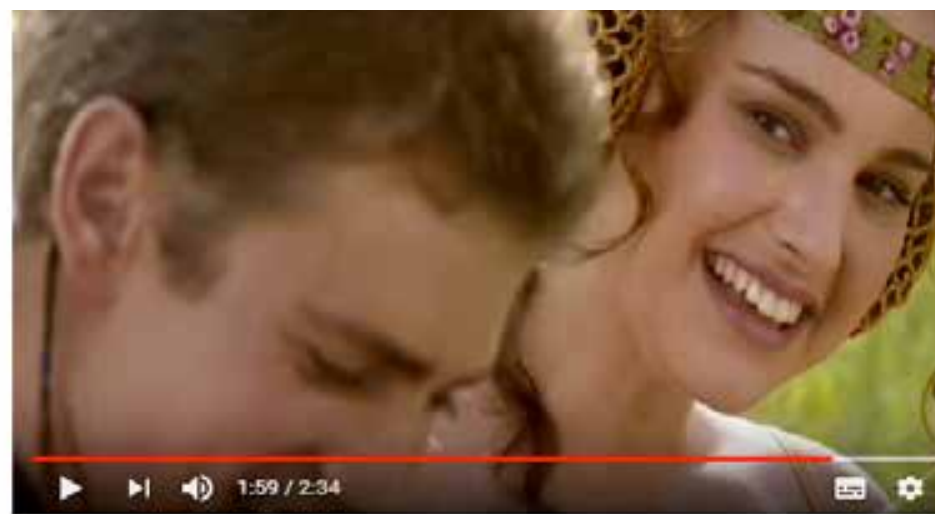

Star Wars / Garden State - Trailer Mash-Up Re-Cut

17873 vues

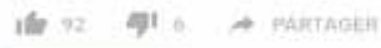

withonea

A putite le 7 levi 2009

Figure 7 : Réactualisation de bandes-annonces de la saga Star Wars par des mash up ${ }^{13}$

Enfin, certains internautes réactualisent des bandes-annonces de Star Wars en concevant de nouvelles versions intégrant (directement sur les images) différents commentaires et décryptages. 


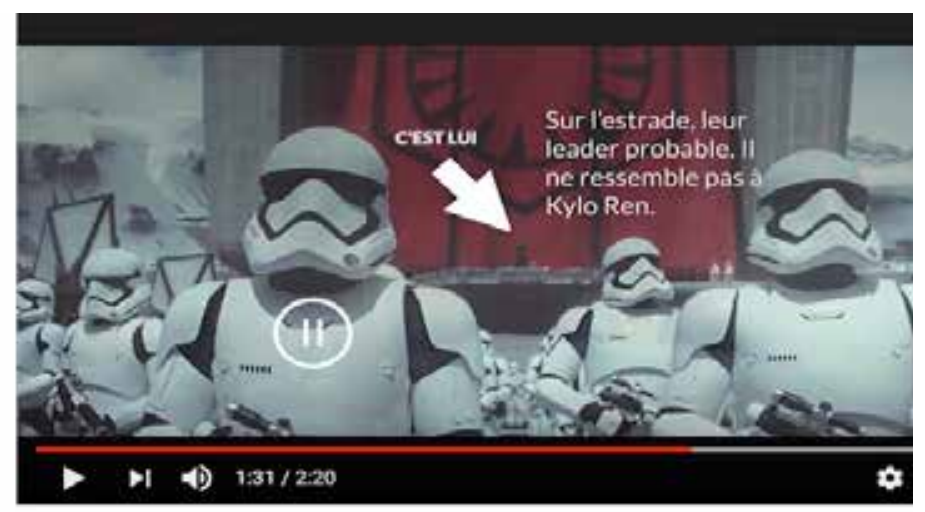

Star Wars VII - Le trailer décrypté

708 vues

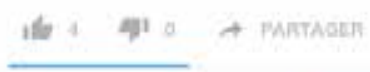

(IIB Ijsberg

Ajoutce le thation, 2015

Figure 8 : Réactualisation de bandes-annonces de la saga Star Wars par des vidéos-décryptages ${ }^{14}$

Nous avons également repéré d'autres pratiques consistant à réactualiser des bandes-annonces de Star Wars en les recréant intégralement. En effet, certains internautes reconstruisent intégralement certaines teasers de Star Wars en se mettant en scène personnellement et en reproduisant les scènes, les décors et les costumes, dans la mouvance des cosplay, c'est-à-dire des pratiques consistant à jouer le rôle d'un personnage en imitant son costume, sa coupe de cheveux, son maquillage. Dans les captures d'écran présentes dans la figure $n^{\circ} 9$, les internautes donnent lieu à des parodies de bandes annonces de la saga. 


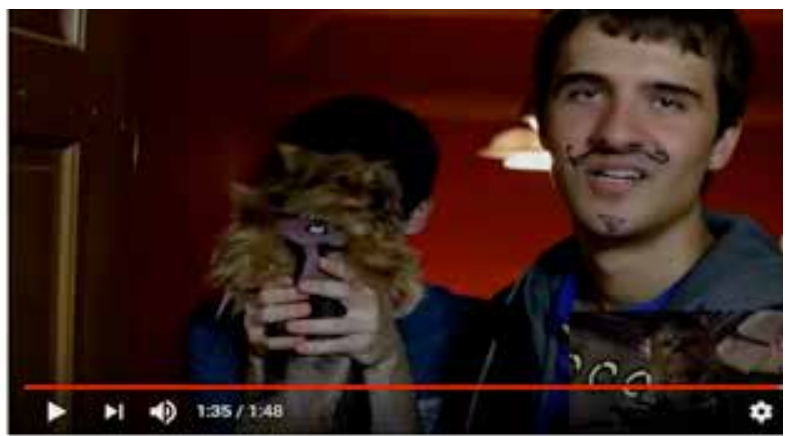

NEW STAR WARS: THE FORCE AWAKENS TRAILER (FANMADE PAROI 350 vues

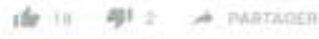

G.7. White on aice

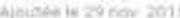

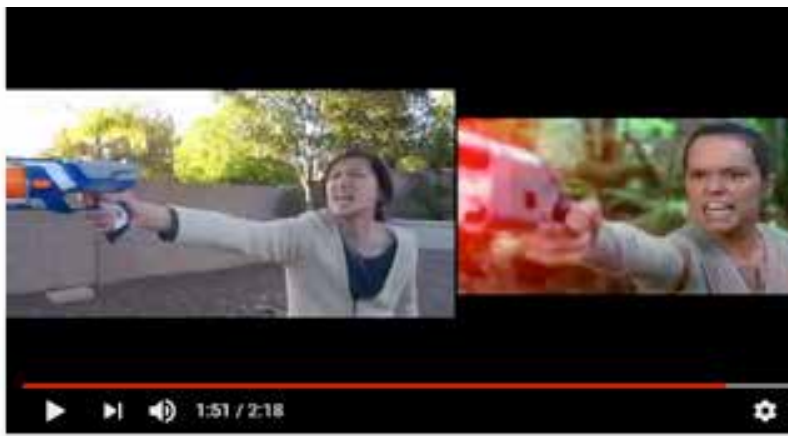

Star Wars: The Force Awakens (Homemade Side by Side Trailer)

11179 vues

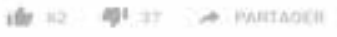

Fortune Cookie Productions

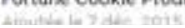

Figure 9 : Réactualisations de bandes-annonces de Star Wars par des parodies

D'autres reconstituent intégralement les bandes-annonces en les recréant sous forme de films en stopmotion, technique d'animation qui crée le mouvement par des arrêts et des reprises répétés de la caméra, à l'aide de dessins ou de figurines comme l'illustre la figure $n^{\circ} 10$. 


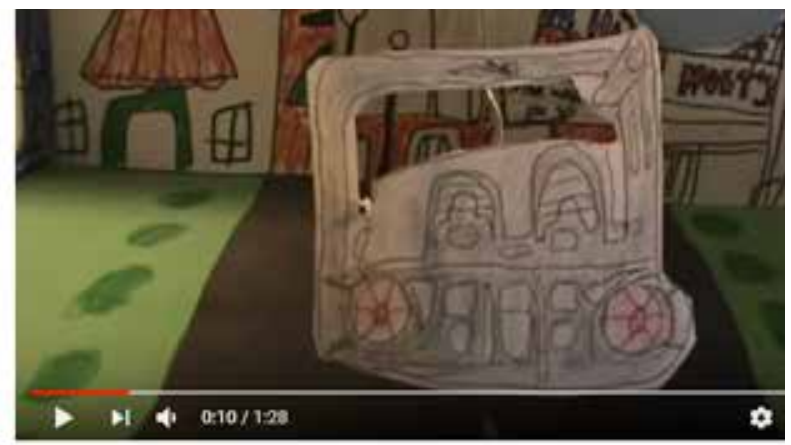

Solo: A Star Wars Story Teaser Trailer Spoof 5055 vees

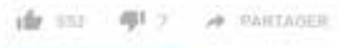

MeHomemadefilms 1

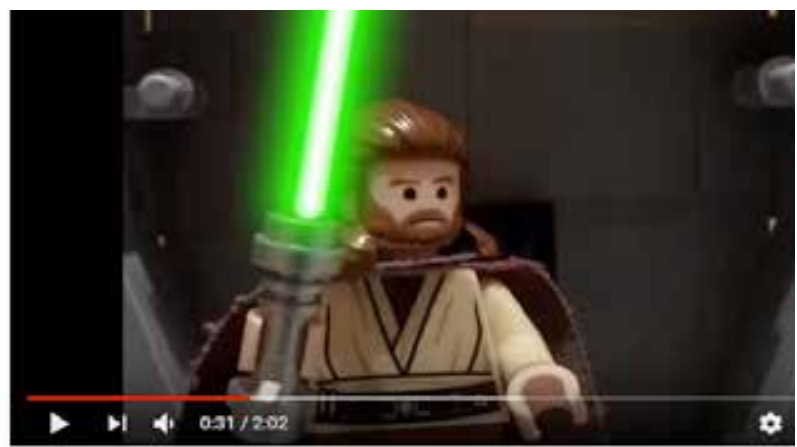

Star wars revenge of the sith trailer in LEGO

7248 wues

Webb studios

Figure 10 : Réactualisations de bandes-annonces de Star Wars par des versions stopmotion

Les pratiques que nous venons de mettre au jour révèlent en quoi les bandes-annonces s'avèrent particulièrement modulables et jouables. En effet, les internautes se les procurent puis les réinventent, les "redocumentarisent » (Pédauque, 2006 ; Salaün, 2007), les « bricolent » (de Certeau, 1990 ; Deuze, 2006) copieusement. Ces pratiques de vidding consistant, pour un amateur, à créer une forme audiovisuelle qui revisite une scène de film ou de série, soulèvent la question de la propriété intellectuelle (Aufderheide et Jaszi, 2008 ; Barthe-Gay, 2010 ; Battisti, 2013) et de la paternité des bandes-annonces bricolées. En effet, 
si ces réactualisations de bandes-annonces témoignent d'un certain nombre de compétences (Auray et Georges, 2012), elles révèlent également un rapport transgressif (Casemajor Loustau, 2012) des internautes aux bandes-annonces, car ces derniers n'attendent pas toujours qu'elles soient libres de droits pour s'en emparer et se les remanier. En ce sens, ces réactualisations des bandes-annonces de Star Wars posent la question du plagiat, par méconnaissance et ignorance du droit (Tabary-Bolka, 2009) ou par mépris et négation des règles éthiques et juridiques de publication et de propriété intellectuelle sur le web. En outre, ces pratiques rappellent que les internautes donnent vie, aujourd'hui, à des circulations transformatives de bandes-annonces, qui posent des questions concernant leur rôle : en quoi les auteurs de ces circulations transformatives peuvent-ils être assimilés à des " bidouilleurs », des "pirates » du numérique (Mattelart, 2011 ; Kyrou, 2015), voire à des ambassadeurs d'une contre-culture?

3.3. Ressuscitation de bandes-annonces anciennes par les internautes : la nostalgie des internautes à l'origine d'une permanence des trailers ?

En étudiant les réactualisations de bandes-annonces de Star Wars initiées par les internautes sur Youtube, nous constatons l'intérêt des internautes pour les bandes-annonces anciennes. En effet, ces derniers ne se contentent pas de réinventer des bandes-annonces récentes, mais s'emploient également à revitaliser des trailers anciens. Cet intérêt pour les anciennes bandes-annonces et cette démarche de " ressuscitation " constituent une manifestation, à notre sens, de l'attention que les internautes - et plus généralement la société contemporaine - accordent aux objets d'un passé récent (Reynolds, 2012 ; Kessous, 2011) : en témoignent notamment l'engouement actuel pour les vinyles, les polaroïds, les consoles old school, les films en noir et blanc. Selon nous, les réactualisations de bandes-annonces que nous avons pointées s'inscrivent dans cette dynamique : elles marquent simultanément l'engouement actuel des internautes pour des objets d'un passé récent et leur capacité à formuler des propositions nouvelles autour de ces héritages. Plus précisément, ces formes de réactualisations peuvent être appréhendées comme des pratiques rétro autour d'objets vintage, parfois teintées de nostalgie ou ancrées dans une esthétique nostalgique (Williams, 2014). En effet, les recyclages que nous avons étudiés esquissent les contours d'une société de la nostalgie : une nostalgie non régressive, créative, ancrée dans la revitalisation d'artefacts publicitaires d'un passé qui n'est pas très éloigné.

Il convient de poursuivre cette réflexion et de s'interroger sur les enjeux portés par ces pratiques de revitalisation de bandes-annonces. Ces formes de réactualisations-ressuscitations ne sont pas sans implications dans l'existence des bandes-annonces. Elles marquent une transformation majeure dans la carrière de ces artefacts publicitaires. En effet, au cinéma, les films ne restent pas à l'affiche ad vitam aeternam. De fait, la promotion d'un film et la durée d'existence des artefacts publicitaires comme les bandes-annonces sont pensées dans une dynamique éphémère. Les professionnels qui les conçoivent et les 
réalisent, les annonceurs qui s'en servent pour faire connaître le film ainsi que les médias qui les diffusent les appréhendent comme des dispositifs éphémères, déployés durant un laps de temps limité. Or, les différentes formes de réactualisations que nous avons repérées invitent à reconsidérer cette fugacité. En effet, celles-ci montrent que les internautes restaurent aujourd'hui des liens avec des bandes-annonces passées et les soustraient de l'existence momentanée et temporaire à laquelle elles étaient destinées. En ce sens, les pratiques que nous avons mises au jour bousculent la carrière des bandes-annonces, et les font basculer de l'éphémère à l'éternel, du temporaire au permanent. Elles donnent vie à un mouvement de résurgence et assurent tout à la fois la survivance et la permanence de bandes-annonces passées, dont la fonction marchande initiale était achevée, et qui n'étaient pas nécessairement destinées à durer après avoir atteint leurs objectifs promotionnels et commerciaux. Ainsi, les pratiques étudiées montrent combien le numérique reconfigure l'existence des trailers passés et permet à des bandes-annonces anciennes de ressurgir. Bien sûr, ce phénomène ne concerne pas exclusivement les bandes-annonces, ou la filière cinématographique. Dans différentes filières (dont celles de la musique et de l'édition), la nostalgie envers certains contenus analogiques a stimulé l'apparition de remédiations et donné progressivement vie à une société du "re " (re-make, re-mix, re-lecture, re-boot, re-mastering, re-créations...) (Détré, 2015) : une société dans laquelle les opportunités offertes par le numérique a progressivement permis la réminiscence, voire la permanence d'objets culturels du passé. Une société d'anachronismes, dans laquelle le passé se conjugue au présent et où co-existent objets anciens, nouveaux ou new old (Caoduro, Baschiera, 2017). Une société de l'ad vitam, dans laquelle les objets, aussi anciens soient-ils, peuvent prétendre à une relative éternité.

3.4. Réactualisation de bandes-annonces anciennes : les internautes (nostalgiques) à l'origine de nouvelles stratégies de marketing?

Si les pratiques numériques nostalgiques que nous avons pointées peuvent être à l'origine d'un phénomène de résurgence et de permanence des bandes-annonces, elles peuvent également être exploitées par un certain nombre de stratégies initiées par les professionnels chargés de la promotion des films. En effet, les pratiques numériques nostalgiques que nous avons étudiées peuvent devenir d'autant plus stratégiques pour les acteurs économiques, qu'elles s'inscrivent dans une dynamique bénévole et virale. Certains professionnels semblent avoir saisi l'engouement des internautes pour les objets du passé (Lizardi, 2017) et le potentiel inhérent à cette nostalgie et développent des stratégies centrées sur ces « internautes nostalgiques ». Les acteurs chargés de la promotion de Star Wars, par exemple, utilisent les médias pour accéder virtuellement au passé (Schrey, 2014), remettre en circulation des contenus anciens (extraits de la saga, artefacts publicitaires...) et inviter les internautes à créer ou du moins à réagir autour de ces contenus. En 2016, ils ont notamment invité les internautes à produire des contenus sur la saga en lançant Les Awards du fanfilm Star Wars. 
D'une part, ces pratiques rappellent que les internautes deviennent parfois des outils de promotion (Bourdaa, 2016) et sont utilisés dans des stratégies de community management (Galibert, 2014), dans lesquelles ils sont invités à converger (Jenkins, 2006 ; Laurichesse, 2015), à côté des professionnels, pour la promotion des films. Cette sollicitation des internautes introduit les questions de marchandisation des communautés en ligne et de transaction de leur attention (Kessoux, 2012). Elle relance, en outre, des questions relatives à la spontanéité de la participation en ligne (Stenger, Coutant, 2015 ; Beauvisage, Mellet, 2016) et à l'authenticité de l'e-réputation des films.

D'autre part, ces pratiques montrent que des stratégies semblent se développer, en ligne, autour de la nostalgie (Niemeyer, 2014) : des stratégies de rétro-marketing, alliant la nostalgie et l'innovation chères aux consommateurs contemporains ${ }^{15}$, exploitant et jouant sur le spectre des manifestations de la now-stalgia (nostalgie contemporaine) (Menke, 2016). Ces pratiques montrent que la nostalgie peut être un phénomène médié, impulsé par des injonctions à la nostalgie ou des stratégies de marchandisation du passé.

\section{Conclusion}

La présente réflexion met au jour différentes formes de réactualisations de bandes-annonces de la saga Star Wars, impulsées par les internautes sur Youtube. Ces réactualisations révèlent une reconfiguration considérable du rapport des publics contemporains aux artefacts publicitaires : un rapport à la fois facilité, décomplexé, et transgressif, dépassant désormais bien souvent l'attention contemplative. Mais ces réactualisations révèlent également combien le numérique transforme les artefacts publicitaires, leur nature (poreuse, modulable, jouable) et leur carrière (étendue et prolongée par les internautes). En outre, les réactualisations de bandes-annonces du passé rappellent l'importance que les individus accordent aujourd'hui aux objets du passé et montrent que leur nostalgie (non régressive, créative) participe à un phénomène de permanence ou de résurgence de certains artefacts qui n'étaient initialement pas voués à traverser les époques. Enfin, les réactualisations de bandes-annonces que nous avons étudiées révèlent le caractère stratégique des « internautes nostalgiques ", aujourd'hui capables de bricoler des artefacts du passé et d'étendre, ainsi, leur existence, jusque dans le présent de nos sociétés.

15 Enquête IPSOS, Marketing : l'effet rétro, disponible sur : https://www.ipsos. $\mathrm{com} / \mathrm{fr}$-fr/marketing-leffet-retro (page consultée le 1er octobre 2018). 
Références bibliographiques

Aufderheide P., Jaszi P. (2008). Recut, Reframe, Recycle: Quoting Copyrighted Material in User-Generated Video, Center for Social Media, Washington.

Auray N., Georges F. (2012). « Les productions audiovisuelles des joueurs de jeux vidéo : Entre formation professionnelle et apprentissages esthétiques autodidactes ". In Réseaux, vol. 175, p. 145-173.

Bardin L. (2013). L'analyse de contenu, Paris, Presses Universitaires de France.

Barthe-Gay C. (2010). « Droit d'auteur et numérique : un équilibre introuvable? ». In Journée d'étude Appropriations et réappropriations de biens culturels à I'heure du numérique, Toulouse.

Battisti M. (2013). " Mash up, remix, sample, machinima... au risque du droit d'auteur? ». In De ligne en ligne, vol. 11, avril-septembre, p. 18-19.

Beauvisage T., Mellet K. (2016). "Travailleurs du like, faussaires de l'e-reputation ". In Réseaux, vol. 197-198, p. 69-108.

Berthelot-Guiet K. (2013). Paroles de pub : la vie triviale de la publicité, Paris, Non Standard.

Beuscart J., Mellet K. (2012). Promouvoir les œuvres culturelles : Usages et efficacité de la publi- cité dans les filières culturelles, Paris, Ministère de la Culture.

Bourdaa M. (2013). « La construction d'un univers de marque à travers des stratégies transmédia : le cas de Hunger Games ». In Laurichesse H. (dir.), La stratégie de marque dans l'audiovisuel, Paris, Armand Colin, p. 43-56.

Bourdaa M. (2016). « La promotion par les créations des fans ». In Raisons politiques, $n^{\circ}$ 62, p. 101-113.

Bourgeon-Renault D. (2009). Marketing de l'art et de la culture, Paris, Dunod.

Caoduro E., Baschiera S. (2017). "The New Old: Archaisms and Anachronisms across Media ». In Alphaville: Journal of film and screen media, vol. 12, p. 1-7.

Casemajor Loustau N. (2012). « La participation culturelle : encadrement et appropriations transgressives du patrimoine numérisé ». In Communication et langages, vol. 171, p. 81-98.

Certeau (de) M. (1990). L'invention du quotidien, Paris, Gallimard.

Charest F., Gauthier A. M., Grenon F. (2013). « Appropriation et stratégies d'intégration des médias sociaux par les professionnels de la communication ». In Communication et organisation, vol. 43, p. 269-280.

Chevry-Pébayle E., De Iulio S. (2015). "Les collections numérisées d'affiches publicitaires : entre construc- 
tion de l'offre et appropriations ». In Les Enjeux de l'information et de la communication, vol. 16, p. 41-52.

Chirouze A. (2007). " Cinéma et publicité : un mariage d'amour ou de raison? ". In Market Management, vol. 7, p. 133-160.

Cochoy F. (2011). De la curiosité. L'art de la séduction marchande, Paris, Armand Colin.

Courbières C. (2016). « De la fonction au mythe ? La K7 audio, retour vers le futur ». In Colloque Du rétro au néo : entre nostalgie et réinvention. Objets en mouvement, Mons, 8-9 décembre.

Croquet C. (1998). « Les processus de médiation et de médiatisation au cours des campagnes de communication des films ». In Études de communication, vol. 21, p. 83-95.

Deaville J. (2016). « Recut and re-tuned: Fan-produced parody trailers ". In The Journal of Fandom Studies 4, vol. 2, p. 209-223.

Détré N. (2015). «RE comme REprise, REmake, REenactment, REproduction, REmix, REéditing, RElecture etc ». In Journée d'études Ré-emprunt, remix et ré-editing dans les arts actuels, Toulouse, 19 novembre.

Deuze M. (2006). « Participation, Remediation, Bricolage : Considering Principal Components of a Digital Culture ». In The Information Society, vol. 22, $n^{\circ} 2$, p. 63-75.

Duchet C. (2009). « Le cinéma et ses nouvelles pratiques promotionnelles ». In CIR-

CAV, vol. 20, p. 179-192.

Euzeby F., Martinez C. (2004). « La bande-annonce cinématographique : quel impact sur la décision d'aller voir le film ? Une étude exploratoire ". In Décision Marketing, vol. 33, p. 39-50.

Ferrandi J., Kruger A. (2009). "L'importance et la confiance accordées aux sources d'informations ». In Management \& Avenir, vol. 30, n²10, p. 165-182.

Galibert O. (2014). « Approche communicationnelle et organisationnelle des enjeux du Community Management ". In Communication \& Organisation, vol. 46, p. 265-278.

Gayraud A., Heuguet G. (2015). « De I'industrie musicale à la rhétorique du service : YouTube, une description critique ". In Communication \& langages, vol. 184, $\mathrm{n}^{\circ} 2$, p. 101-119.

Hartwig L. (2012). "You'll Never See This on the Silver Screen: The Film Trailer as a Template for the Appropriation and Transformation of Hollywood Movies ». In Film Remakes, Adaptations and Fan Productions, Palgrave Macmillan, Basingstoke, p. 215-230.

Jeanneret Y. (2014). Critique de la trivialité. Les médiations de la communication, enjeu de pouvoir, Paris, Non Standard.

Jenkins H. (2002). « The poachers and the stormtroopers: cultural convergence in the Digital Age ». 
In Le Guern P. (dir.), Les Cultes médiatiques. Culture fan et œuvres cultes, Rennes, Presses Universitaires de Rennes, p. 343-378.

Jenkins H. (2006). Convergence culture, where old and new media collide, New York University Press.

Kessous A. (2011). « Impact de la nostalgie sur les relations des consommateurs aux marques ". In Management \& Avenir, vol. 48, p. 423-438.

Kessoux E. (2012). « Le marketing des traces et la transaction des attentions ». In Kessoux E. (dir.), L'attention au monde : sociologie des données personnelles à l'ère numérique, Paris, A. Colin, p. 59-76.

Kyrou A. (2015). «Eloge des bandits du numériques $»$. In Nectart, vol. 1, p. 118-127.

Laurichesse H. (2009). « La publicité pour le cinéma ». In CIRCAV, vol. 20, p. 163-179.

Laurichesse H. (2015). « (Re)penser les publics de l'industrie cinématographique dans la culture de la convergence ". In RFSIC, vol. 7. Disponible sur https://journals. openedition.org/rfsic/1507 (page consultée le $1^{\text {er }}$ octobre 2018).

Lizardi R. (2017). Nostalgic Generations and Media. Perception and Time of Available Meaning, London, Lexington Books.

Lozano Delmar J. (2012). «Transformaciones comunicativas en el consumo del tráiler cinematográ- fico en internet ». In Questiones

Publicitarias, vol. 17, p. 94-109.

Marion G. (2003). « Le marketing expérientiel : Une nouvelle étape? Non, de nouvelles lunettes ». In Décisions Marketing, vol. 30, p. 87-91.

Mattelart T. (2011). Piratages audiovisuels. Les voies souterraines de la mondialisation culturelle, Paris-Bruxelles, Ina-De Boeck.

Menke M. (2016). « Media, Communication and Nostalgia ». In Medien\&zeit, vol. 31, $n^{\circ} 4$. Disponible sur https : //medienundzeit.at/category/ jahrgang-2016-31/heft-2016-4/ (page consultée le 1er octobre 2018).

Niemeyer K. (2014). Media and Nostalgia. Yearning for the Past, Present and Future, Houndmill, Palgrave.

Pédauque R. (2006). Le document à la lumière du numérique : forme, texte,médium, C\&F Éditions.

Reynolds S. (2012). Rétromania, Comment la culture pop recycle son passé pour s'inventer un futur, Marseille, Le Mot et le Reste.

Salaün J. M. (2007). « La redocumentarisation, un défi pour les sciences de l'information ". In Études de communication, vol. 30, p. 13-23.

Schrey D. (2014). «Analogue Nostalgia and the Aesthetics of Digital Remediation ». In Niemeyer K. (dir.), Media and Nostalgia. Yearning for the Past, Present, and Future, Basingstoke, New York, p. 27-38. 
Stenger T., Coutant A. (2015). « L'appropriation des réseaux socio-numériques et les arts de l'encadrer : pour une prise en compte des rapports de force entre utilisateurs et dispositifs ". In Systèmes d'information et management, vol. 20, p. 89-122.

Tabary-Bolka L. (2009). « Culture adolescente vs culture informationnelle. L'adolescent acteur de la circulation de l'information sur internet ». In Les Cahiers du numérique, vol. $5, n^{\circ} 3$, p. 85-97.

Williams K. (2010). «Re-reading the history of the trailer: The production and consumption of "recut" trailers». In Mc Callum K. (dir.), Australian and New Zealand Communication Association Conference, Canberra, ANZCA. Disponible sur https://www.anzca.net/ documents/2010-conf-papers/460re-reading-the-history-of-the-trailerthe-production-and-consumptionof-recut-trailers-1/file.html (page consultée le 1er octobre 2018).

Williams K. (2014). « Recut Film Trailers, Nostalgie et le film Teen film ». In Barton K., Lampley J. (dir.), Fan culture, McFarland \& Company, Jefferson, p. 47-59. 